\title{
Personalised medicine in asthma: from curative to preventive medicine
}

\author{
Laurent Guilleminault ${ }^{1,2,3}$, Hakima Ouksel ${ }^{4}$, Chantal Belleguic ${ }^{5}$, \\ Yannick Le Guen ${ }^{5,6}$, Patrick Germaud ${ }^{7}$, Emilie Desfleurs ${ }^{8}$, Christophe Leroyer $^{9}$ \\ and Antoine Magnan 7,10
}

\begin{abstract}
Affiliations: 'Dept of Pulmonary Medicine, Reunion Island University Hospital/South Reunion Island Hospital Group, Saint-Pierre, France. ${ }^{2}$ INSERM, UMR 1188 Diabetes-Atherothrombosis Therapies Reunion Island Indian Ocean (DéTROI), Reunion Island Indian Ocean Cyclotron (CYROI) Platform, Sainte-Clotilde, France. ${ }^{3}$ University of Reunion Island, UMR 1188, Sainte-Clotilde, France. ${ }^{4}$ Dept of Pulmonary Medicine, Angers University Hospital, Angers, France. ${ }^{5}$ Dept of Pulmonary Medicine, Rennes University Hospital, Rennes, France. ${ }^{6}$ Pulmonary Medicine Practice, St Grégoire Private Hospital, Saint-Grégoire, France. ${ }^{7}$ Nantes University Hospital, Nantes-Roscoff National Cystic Fibrosis Reference Centre, Nantes, France. ${ }^{8}$ Novartis Pharma, Scientific Operations, Rueil-Malmaison, France. ${ }^{9}$ European University of Brittany, University of Brest, EA3878, IFR148, Dept of Internal and Respiratory Medicine, La Cavale Blanche Hospital, Brest, France. ${ }^{10}$ UMR_S 1087 CNRS UMR_6291, L'Institut du Thorax, University of Nantes, Nantes, France.
\end{abstract}

Correspondence: L. Guilleminault, Service de Pneumologie, CHU Réunion/GHSR, BP 350, 97448 Saint-Pierre cedex, F-97410, France. E-mail: laurent.guilleminaultachu-reunion.fr

@ERSpublications

The personalised curative approach in asthma should make way for personalised preventive and predictive medicine http://ow.ly/4nfq $2 \mathrm{w}$

Cite this article as: Guilleminault L, Ouksel H, Belleguic C, et al. Personalised medicine in asthma: from curative to preventive medicine. Eur Respir Rev 2017; 26: 160010 [https://doi.org/10.1183/16000617.00102016].

ABSTRACT The concept of asthma has changed substantially in recent years. Asthma is now recognised as a heterogeneous entity that is complex to treat. The subdivision of asthma, provided by "cluster" analyses, has revealed various groups of asthma patients who share phenotypic features. These phenotypes underlie the need for personalised asthma therapy because, in contrast to the previous approach, treatment must be tailored to the individual patient. Determination of the patient's asthma phenotype is therefore essential but sometimes challenging, particularly in elderly patients with a multitude of comorbidities and a complex exposure history. This review first describes the various asthma phenotypes, some of which were defined empirically and others through cluster analysis, and then discusses personalisation of the patient's diagnosis and therapy, addressing in particular biological therapies and patient education. This personalised approach to curative medicine should make way in the coming years for personalised preventive and predictive medicine, focused on subjects at risk who are not yet ill, with the aim of preventing asthma before it occurs. The concept of personalised preventive medicine may seem a long way off, but is it really?

\section{Introduction}

Life expectancy is increasing worldwide and especially in Europe, and this is certainly good news. And yet increased life expectancy means an ageing population, more dependence and more disability. It will also bring increased prevalence of chronic diseases in general, and in particular of patients with respiratory disabilities, including asthma. Faced with the prospect of rising healthcare costs due to the ageing population, and bearing in mind the current economic crisis in Europe, this news is also rather worrying.

Received: Feb 172016 | Accepted after revision: April 152016

Conflict of interest: Disclosures can be found alongside this article at err.ersjournals.com

Provenance: Submitted article, peer reviewed.

Copyright $\odot$ ERS 2017. ERR articles are open access and distributed under the terms of the Creative Commons Attribution Non-Commercial Licence 4.0. 
Medical care could take an alternative course in the coming years, however. Given developments in predictive techniques, instead of concentrating most of our spending on curative treatments, we could focus on healthy subjects at risk and allocate sufficient resources to preventive medicine.

Recent years have seen an extremely rapid rise in biotechnologies. With high-throughput sequencing and the widespread availability of molecular biology analysis at the individual-patient level, it will soon be possible to take a drop of blood from a person's fingertip and have their entire genome sequenced in under an hour. These new health technologies will facilitate the development of preventive medicine and the use of the targeted therapies now being developed for chronic diseases, as well as for cancer. Asthma is no exception, with the advent of omalizumab a few years ago, and anti-cytokine and anti-cytokine receptor antibodies more recently. The use of these biologics in clinical practice and in phase 3 studies has revealed the existence of responders and nonresponders. The heterogeneity in the responses observed to these targeted treatments reinforces the notion of asthma phenotypes and subdivides this common disease into a multitude of rarer diseases. Detailed analysis of gene expression in responders will make it possible to identify specific biomarkers for the targets of these advanced therapies, thereby enabling their development.

This review, prepared by specialists in severe asthma, starts by outlining how asthma is viewed today and how its management is already marked by a certain degree of personalisation. The implications of this view should lead us to reconsider our management of asthma, with the development of biotechnologies and biological therapies specifically for asthma. The change will not only affect the drugs we prescribe, but more generally, will lead to a holistic approach to our patients, involving 4P (preventive, predictive, personalised and participative) medicine.

\section{Asthma phenotypes}

\section{The general concept}

Asthma is a heterogeneous entity comprising several diseases. The concept of subdividing asthma into different diseases emerged in the late 1990s [1]. The idea of asthma phenotypes originated from this desire to define subgroups of patients with shared clinical features in order to better understand asthma pathophysiology and improve its treatment [2]. The greatest contribution came from the use of mathematical algorithms, which replaced the subjective approach previously used by clinicians [3]. These multivariate algorithms defined asthma phenotypes by objectively grouping patients with similar clinical features into clusters $[4,5]$.

HaLDAR et al. [6] applied this method to patients with mild-to-moderate asthma managed in primary care and patients with severe asthma managed in secondary care. The variables used in the algorithm were symptoms, atopy/allergy, eosinophilic inflammation, psychological status and airflow obstruction. Other variables included sex, body mass index (BMI) and age of asthma onset. For mild-to-moderate asthma managed in primary care, these authors demonstrated three asthma subtypes: early-onset atopic asthma; obese, predominantly female asthma without eosinophilic airway inflammation; and "benign" asthma with little evidence of active disease. The early-onset atopic asthma subgroup and the obese, predominantly female subgroup were also identified among patients managed in secondary care. Two other subgroups were demonstrated: patients with high symptom expression, early onset and minimal eosinophilic inflammation, and patients with predominant eosinophilic inflammation, few symptoms, late onset and male preponderance.

Different sets of phenotypes are defined depending on the variables used. In a different study in patients with severe asthma, 34 variables were used [7]. Five patient subgroups were defined. The results obtained resembled those of the aforementioned study but the subgroup definitions were more detailed due to the greater number of variables used. Subgroup 1 comprised predominantly female patients with early-onset atopic asthma and normal lung function, requiring little medication. Subgroup 2 included subjects with early-onset asthma and preserved lung function, and were predominantly female but used three or more drugs. Subgroup 3 was characterised by older, obese women with late-onset asthma. Their forced expiratory volume in $1 \mathrm{~s}(\mathrm{FEV} 1)$ was moderately decreased and oral corticosteroid use was common. Subgroups 4 and 5 both had airflow obstruction. Subgroup 4 patients were atopic with childhood-onset asthma; both sexes were equally represented. Subgroup 5 patients differed from subgroup 4 patients in the late onset of their disease, female preponderance and less atopy. These results have been confirmed in other studies $[8,9]$. A patient's phenotype appears to remain relatively stable over time [10]. The choice of variables used in cluster analyses is crucial. Although these studies have greatly improved the understanding of asthma, their results probably present a simplified and simplistic view of the disease. It is currently difficult to incorporate comorbidities and other complex factors such as diet and exposure history into these studies.

\section{Features of the various asthma phenotypes}

Asthma and allergy

Early-onset asthma, where symptoms begin in childhood, seems to have an allergic mechanism [11]. Compared with nonallergic asthma, allergic asthma also exhibits seasonal variation related to allergen exposure [11]. 
Total IgE levels seem to be higher in allergic than in nonallergic asthma but this feature does not in itself define an allergic asthma phenotype [12]. Blood or sputum eosinophilia is observed in both allergic and nonallergic asthma [13]. Allergen exposure leads to an increase in exhaled nitric oxide fraction ( FeNO) levels but does not constitute a marker for allergic asthma. It is, however, a marker for airway eosinophilia [14]. Allergen sensitisation must be investigated systematically, given the therapeutic implications.

\section{Asthma and obesity}

An obese asthma phenotype has been identified in adults in cluster analyses [6,7]. It is typically described as late-onset asthma with little allergy, frequent symptoms, noneosinophilic inflammation and high medication use [7, 15-18]. In the paediatric population, a phenotype of early-onset asthma associated with obesity has been identified [19]. In this group, asthma symptoms begin before 12 years of age and the sex ratio is equal. This phenotype is atopic in nature, with elevated IgE levels. These patients also exhibit severely decreased airway function, significant airway hyperresponsiveness and poor asthma control.

The link between obesity and asthma is probably more complex than the view offered by cluster analysis. In these studies, noneosinophilic inflammation is typically observed in adult patients based on sputum assessment. However, another study has shown that sputum interleukin (IL)-5 and submucosal eosinophils, but not sputum eosinophils, are elevated in obese patients with severe asthma [20]. A multitude of factors could explain the mechanism of asthma in obese patients. It includes changes in lung structure and function (reduced lung volume increases airway reactivity), oxidative signalling, cytokine derangement and neuronal signalling pathways [21]. Diet has also to be taken into account in obese patients with asthma due to its key role in this group. Indeed, it has been demonstrated that a pro-inflammatory diet (such as a high-fat diet) increases airway inflammation in asthma patients, particularly in those with obesity [22]. Comorbidities such as gastro-oesophageal reflux disease, obstructive sleep apnoea and depression are very common in obese patients and can worsen asthma outcome [23]. Asthma in obese patients is a complex entity that requires personalised management.

\section{Fungal hypersensitivity}

Two phenotypes are linked to fungi: asthma associated with fungal hypersensitivity and allergic bronchopulmonary mycoses. The predisposing factors for these phenotypes are both genetic and environmental. Polymorphisms in genes encoding Toll-like receptors, chitinases (24-bp duplication of the CHIT1 gene), and major histocompatibility complex HLA-DR 2 and 5 may play a role [24, 25]. Exposure to moulds in the home, in the workplace or outdoors (for example, thunderstorm asthma) plays a role in asthma development and exacerbations.

Asthma associated with fungal hypersensitivity can be moderate. However, it often progresses to severe asthma, especially when the patient is exposed to abnormally high fungal concentrations [26, 27]. Severe asthma with fungal sensitisation (SAFS) was defined in 2006 by the combination of three features: the presence of severe asthma; fungal sensitisation, determined by prick testing or specific IgE testing; and total $\mathrm{IgE}$ of $<1000 \mathrm{IU} \cdot \mathrm{mL}^{-1}$ [28]. SAFS sometimes progresses to allergic bronchopulmonary mycosis, the main features of which are the same as in SAFS accompanied by total IgE levels $>1000 \mathrm{IU} \cdot \mathrm{mL}^{-1}$.

The treatment of severe forms is based on high doses of an inhaled corticosteroid in combination with a long-acting inhaled $\beta_{2}$-agonist and systemic corticosteroid therapy. Omalizumab or itraconazole may improve asthma control but there are no formal guidelines on their use [29, 30]. The possibility of environmental risks in the home or workplace should be investigated systematically, ideally with an intervention by an indoor environment consultant, with the aim of reducing exposure to asthma triggers.

\section{Exercise-induced bronchospasm}

Some phenotypes were recognised long before cluster analyses existed, such as exercise-induced bronchospasm [31]. Symptoms develop after a brief period of exercise and can last for 30-90 min if untreated [32]. Its prevalence varies from $30 \%$ to $70 \%$, depending on the population studied [33]. A key diagnostic criterion is a fall in FEV1 of $10 \%$ or more after exercise [34]. In clinical practice, the diagnosis is based on the patient interview. Recommended treatments are short-acting $\beta_{2}$-agonists taken 15 min before exercise, leukotriene receptor antagonists or inhaled corticosteroids [35]. 
Asthma-chronic obstructive pulmonary disease overlap syndrome

Asthma-chronic obstructive pulmonary disease (COPD) overlap syndrome (ACOS) is an entity characterised by airflow limitation, and shares some features from asthma and COPD [37]. The prevalence of ACOS among adults with asthma is $20.9 \%$, ranging from $5.2 \%$ to $35.4 \%$ [38]. ACOS is a heterogeneous entity and several ACOS phenotypes have already been identified, such as asthma in smokers and neutrophilic ACOS [39]. However, the description of ACOS is still ongoing and the treatment strategy will probably change in the future with a better understanding of ACOS phenotypes. For now, in the absence of specific studies of ACOS, its treatment is extrapolated from phenotype-directed specific treatments for severe asthma and COPD. Some of the personalised therapies discussed below could be used in ACOS phenotypes that are similar to asthma phenotypes.

\section{Asthma in the elderly}

The clinical features of asthma in the elderly are very complex and quite distinct from the disease in young adults [40]. The prevalence of asthma in the elderly ranges from $4.5 \%$ to $12.7 \%$, and includes both longstanding early-onset asthma and adult-onset disease [41, 42]. The clinical syndrome among elderly asthma patients is very heterogeneous, and frequently co-exists with a combination of comorbidities and phenotypes, such as obesity, COPD, depression, atopy (less commonly) and a complex exposure history. Moreover, poor adherence is common among elderly asthma patients [43]. For all these reasons, the personalised approach detailed here is necessary when treating asthma in the elderly to improve outcomes.

\section{Some limitations of the phenotypic approach}

In a proportion of cases, the phenotypic approach has certain limitations. Several phenotypes may be observed in a single patient, making phenotype-specific management very difficult. Furthermore, it has been reported that the various phenotypes within severe asthma do not differ in asthma control-related outcomes [44]. The phenotypic approach is, for now, unable to predict asthma control outcomes on which the response to innovative therapies is assessed.

\section{Phenotypic approach to asthma assessment}

In order to classify patients into asthma subgroups based on cluster analyses, some clinical criteria are essential $[6,7]$. Age, sex and BMI are key criteria in the process of phenotyping asthma patients. As an example, the "obese women" phenotype is frequently identified in studies [6, 7]. Smoking status does not in itself appear to define asthma phenotype [45-47]. It is, however, responsible for an increased risk of exacerbation in children and adults, and impairs lung function [48-50]. The age of asthma onset must also be determined. Early onset will guide the diagnosis towards atopic phenotypes. The patient should also be questioned about any situations that trigger asthma symptoms, such as exercise. Patients should be systematically asked whether aspirin is a trigger because of the specific treatment considerations that apply to the aspirin-induced phenotype [51]. The number of exacerbations per year is an important variable, partly to identify poor asthma control but also to classify the patient as a frequent exacerbator requiring a step up in therapy.

Atopic asthma is the commonest asthma phenotype [7]. It is important to investigate the patients' home and work environments to find out whether they are exposed to any allergens, or irritant or toxic substances, possibly through an intervention by a medical indoor environment consultant [52]. Allergy testing is based on skin-prick tests, guided by the patient's reported allergic history. It is difficult to list all the allergens to be tested but the tests recommended in Europe are for the allergens from the GA ${ }^{2} \mathrm{LEN}$ (Global Allergy and Asthma European Network) study (table 1) [53].

Spirometry is essential in the management of asthmatic patients. Airflow obstruction with an FEV 1 of $<80 \%$ of the predicted value is an important feature, as it defines more severe patient phenotypes in which a step up in therapy could be considered [7]. The persistence of bronchodilator reversibility does not constitute a phenotype in itself but is more frequent in atopic subgroups [7]. Bronchodilator reversibility is less common among obese patients, including children [54]. FeNO is traditionally defined as a marker of bronchial and alveolar eosinophilia [55]. Its value in asthma is much debated and there is currently no evidence to support its use in the differential diagnosis of asthma phenotypes [56].

Few laboratory and radiological investigations are of relevance to these assessments, in the absence of more effective biomarkers, and are only worth considering in severe asthma. Blood and sputum eosinophil counts can be helpful in patients with severe asthma because the eosinophilic asthma phenotype, found in cluster analyses, tends to be severe and marked by frequent exacerbations. Total IgE is only worth measuring if allergic bronchopulmonary mycosis is suspected or before initiating omalizumab therapy [57]. A chest computed tomography scan should be performed to look for central bronchiectasis, which would suggest allergic bronchopulmonary mycosis [57]. 
TABLE 1 Skin-prick tests recommended by the European Academy of Allergy and Clinical Immunology

Pollens

Animals

House dust mite

Other

\author{
Hazel \\ Alder \\ Birch \\ Plane \\ Cypress \\ Grass mix \\ Olive \\ Mugwort \\ Ragweed \\ Parietaria \\ Alternaria alternata \\ Aspergillus fumigates \\ Cat \\ Dog \\ Dermatophagoides pteronyssimus \\ Dermatophagoides farinae \\ Cockroach (Blattella germanica)
}

Reproduced and modified from [53] with permission.

Pharmacotherapy for severe asthma: the prospect of personalised medicine

The phenotypic approach detailed here is useful in mild-to-moderate asthma as well as in severe asthma. As described in the following section, personalised nondrug therapies, such as education, are offered to all asthma patients but adapted to each one individually. Innovative therapies are currently reserved for severe asthma and based on asthma phenotype.

\section{Allergic phenotype}

Omalizumab is a humanised anti-IgE monoclonal antibody that forms complexes with circulating IgE. It inhibits IgE-mediated responses and downregulates high-affinity IgE receptors on mast cells and basophils.

In the INNOVATE pilot study, compared with placebo, omalizumab reduced the asthma exacerbation rate by $26 \%$ and the rate of severe exacerbations by $50 \%$ in patients with severe allergic asthma and frequent exacerbations (two or more per year). The Cochrane Airways Group's 2013 meta-analysis of 25 studies found that, compared with placebo, omalizumab significantly reduced both the number of exacerbations, with an odds ratio of 0.55 , and the number of hospitalisations, with an odds ratio of 0.16 , in patients with moderate-to-severe asthma [58].

Few adverse effects were reported. The main one was injection site pain in $9.1 \%$ of omalizumab-treated patients versus $5.6 \%$ of patients in the placebo group.

The possibility of rare adverse effects that only emerge after prolonged omalizumab use has also been investigated. In a North American observational study in 7857 patients (5007 receiving omalizumab and 2829 controls), the incidence of malignancy was identical in both arms (hazard ratio (HR) 1.09, 95\% CI $0.87-1.38)$ [59].

The optimal treatment duration has not been determined. A phase 3 study in 176 patients evaluated the benefit of continuing omalizumab beyond 5 years [60]. The group that continued the treatment had fewer exacerbations and better asthma control, while the time to exacerbation was significantly shorter in patients who did not continue receiving omalizumab (HR 0.49, 95\% CI 0.28-0.86). However, many patients who stopped taking the treatment had experienced no exacerbations 1 year after discontinuation, a finding that had already been described in other studies [61].

In a recent meta-analysis that included analysis of the economic impact of omalizumab therapy, six of the seven studies concluded that it was reasonably cost-effective [62]. Two of these studies mentioned that omalizumab was only cost-effective in nonsmokers or responders with the severest asthma.

A North American study estimated that $32.2 \%$ of asthmatic patients do not respond to omalizumab [63]. If personalised medicine is to be achieved, it is essential to identify factors that predict treatment response. A combination of biomarkers of airway inflammation associated with the T-helper cell (Th) type 2 pathway, such as high FeNO, blood eosinophilia and high serum periostin, appears to be associated with omalizumab responder status [64]. Additional studies are required to confirm this result. 


\section{Eosinophilic phenotype}

\section{Anti-IL-5 antibodies}

IL-5 is a major maturation and differentiation factor for eosinophils and represents an interesting therapeutic target in asthma, particularly in phenotypes with blood or sputum eosinophilia [65].

\section{Mepolizumab}

Mepolizumab is a humanised, high-affinity, IgG1, anti-IL-5 monoclonal antibody. The first studies conducted in unselected patients with severe asthma were disappointing [66-69]. An observed reduction in sputum or blood eosinophil counts prompted studies into the effects of mepolizumab specifically in patients with eosinophilic asthma [67-69]. For example, two pilot studies showed that mepolizumab reduced oral corticosteroid use and exacerbations in asthmatic patients with sputum eosinophilia ( $>3 \%$ eosinophils in the sputum sample) $[70,71]$. Other markers of efficacy that are more readily obtained than sputum eosinophil counts, such as the blood eosinophil count, have been investigated. Recent, larger studies have confirmed the value of mepolizumab in asthmatic patients with raised levels of eosinophils in the blood. For example, BEL et al. [72] showed that mepolizumab reduced the dose of oral corticosteroids used in patients with blood eosinophilia by $50 \%$, while no change in corticosteroid use was observed in the placebo group. Eosinophilia was defined as $>300$ eosinophils per microlitre of blood during the previous 12 months or $>150$ eosinophils per microlitre of blood during the optimisation phase. Mepolizumab also reduced the exacerbation rate in asthmatic patients with blood eosinophilia by between $47 \%$ and $53 \%$, depending on the study and the dose used [73, 74]. The effect on exacerbation rate seems even more marked in patients whose blood eosinophil levels are $>500$ cells per microlitre [74].

\section{Reslizumab}

Another anti-IL-5 antibody, reslizumab, is currently under development, with several phase III studies underway. In a study conducted in 2011, this therapeutic antibody did not improve asthma control in patients with severe asthma and sputum eosinophilia ( $>3 \%$ eosinophils), although a trend towards improved scores in the Asthma Control Questionnaire was seen [75]. In a recently published phase III study, reslizumab reduced exacerbations in asthmatic patients with blood eosinophilia ( $>400$ eosinophils per microlitre) who had experienced at least one exacerbation during the previous 12 months [76]. Asthma exacerbations were reduced by $50 \%$ to $69 \%$ with reslizumab compared with placebo (in two distinct studies analysed separately).

\section{Benralizumab}

Benralizumab is a therapeutic antibody that binds to the $\alpha$-chain of the IL-5 receptor [77]. Like other antibodies that target the IL-5 pathway, it is very effective at reducing eosinophils in the airways and sputum [78]. In a phase IIb study, $20 \mathrm{mg}$ and $100 \mathrm{mg}$ benralizumab reduced the number of exacerbations compared with placebo in asthmatic patients with blood eosinophilia (at least 300 eosinophils per microlitre) [79]. Phase III studies are currently in progress to confirm these results.

\section{Therapies that target IL-4 and IL-13}

IL-4 is a cytokine involved in T-cell differentiation and another potential target in asthma therapy [80]. The anti-IL-4 receptor antibody dupilumab reduced exacerbations by $87 \%$ in patients with moderate-to-severe asthma and blood eosinophil counts $>300$ cells per microlitre or with $>3 \%$ eosinophils in sputum [81]. This antibody, the efficacy of which has also been demonstrated in atopic dermatitis, could be particularly useful in patients with pulmonary and cutaneous allergic disease [82]. Pitrakinra, an IL-4 and IL-13 inhibitor, has a moderate effect on allergic asthma [83].

The anti-IL-13 antibody lebrikizumab improves lung function in patients with poorly controlled asthma despite inhaled corticosteroids and appears particularly effective in a subgroup of patients with high blood levels of periostin [84]. Tralokinumab, an anti-IL-13 antibody of the IgG4 subclass, demonstrated no efficacy on asthma control scores but seems to improve lung function [85]. Phase III studies on all these therapeutic antibodies are currently underway.

Innovative therapies such as biologics are, for now, mainly used in patients with severe asthma. For several reasons, we could speculate that, in the future, these drugs would be administered in all forms of asthma. Indeed, production costs may fall in the coming years, making these treatments more accessible and attractive for mild-to-moderate asthma. Moreover, a recent study showed that administration of these treatments can be strategically timed [86]. In this study, pre-seasonal treatment with omalizumab prevented the autumn peak in asthma exacerbations mainly observed in young patients. The key challenge to perfecting curative personalised medicine in asthma will be to refine the use of biologics by defining the optimum time of administration and the subset of patients who respond to the treatment. 


\section{Therapy in other phenotypes}

In the asthma with obesity phenotype, weight loss obtained through appropriate dietary measures and regular exercise has been shown to improve both airway inflammation and asthma control in children and adults [87-90]. Bariatric surgery might be an interesting option for selected patients with severe obesity $[91,92]$. In asthma accompanied by sleep apnoea syndrome, continuous positive airway pressure (CPAP) seems to have a beneficial effect on airway inflammation [93, 94]. Studies are currently underway to determine the effect of CPAP on asthma control in patients with sleep apnoea syndrome.

The noneosinophilic asthma phenotype is a poorly defined entity for which the treatment options are less effective. It is sometimes referred to as the "neutrophilic phenotype" due to the presence of elevated neutrophil numbers in sputum. Macrolide antibiotics have been shown to reduce the rate of severe exacerbations in a subgroup of noneosinophilic asthmatic patients (low FeNO and low blood eosinophil counts), while exhibiting no efficacy in unselected asthmatic patients [95]. In patients with severe asthma in whom neutrophils represented $>40 \%$ of sputum cells, the CXCR2 receptor antagonist SCH527123 reduced the percentage of neutrophils in sputum by $36 \%$, versus a $6 \%$ increase with placebo [96]. A modest decrease in exacerbations was observed in this study. The anti-IL-17 antibody brodalumab appears to have no efficacy on asthma control in patients with moderate-to-severe asthma [97]. Subgroup analyses appeared to show better asthma control than with placebo in patients with high reversibility (post-bronchodilator improvement in FEV1 of $20 \%$ or more).

There is a real need for new and effective therapies for noneosinophilic asthma.

The search for biomarkers in asthma has received much attention in recent decades. In addition to diagnostic biomarkers, predictive biomarkers are needed to determine which patients are likely to respond to expensive therapies. Th2 biomarkers have been widely studied and many of them, such as blood/ sputum eosinophilia, FeNO, serum IgE levels and serum periostin, represent serious candidates [98]. Discrimination between type 2 and non-type 2 inflammation is currently considered the most important way to understand asthma and improve its management. However, despite intensive research, little is known about non-type 2 inflammation and many asthma patients will not benefit from the development of therapies mainly for use in type 2 inflammation. New strategies are needed to improve asthma management, such as the new concept we describe in the next section.

\section{Thermoplasty}

One innovative therapeutic strategy developed in the last decade is bronchial thermoplasty [99]. This method involves heating the bronchial tissue to $65^{\circ}$ with a temperature-controlled radiofrequency catheter, introduced via a flexible bronchoscope. Three divided sessions are required to treat all the bronchi $>3 \mathrm{~mm}$ in diameter, avoiding the middle lobe. The mechanism of action has been partially elucidated in animal and human studies, with demonstration of a reduction in airway smooth muscle mass [100, 101].

The results of several studies are now available. In the international trial Asthma Intervention Research (AIR) that included 112 patients treated with inhaled corticosteroids and an inhaled long-acting bronchodilator, a significant decrease in the number of mild exacerbations, and improvement in quality of life scores and asthma control were observed in the thermoplasty group compared with the control group [102]. The double-blind randomised trial AIR2 in 288 patients with moderate-to-severe, uncontrolled asthma was particularly interesting because it compared thermoplasty with a sham endoscopic procedure [103]. After 1 year of follow-up, a significant improvement in the Asthma Quality of Life Questionnaire score and a reduction in the rate of hospitalisations for asthma were observed in the thermoplasty group compared with the placebo group. In this trial, as in other, smaller studies, no improvement in lung function was observed [100]. In these studies, all the patients had a baseline FEV1 greater than or equal to $60 \%$ of the predicted value. Do the data indicate which patients should be eligible for this therapeutic approach? CHANEz et al. [104] have proposed that the patients most likely to benefit from bronchial thermoplasty would be those with bronchial "lability", as evidenced by a good response to bronchodilators and a tendency toward exacerbations. The current approach has been to establish a cohort of patients with a more severe profile than this, described as severe "refractory" asthma (www.clinicaltrials.gov identifier number NCT01185275). Various biomarkers will be analysed in this cohort with the aim of defining which subgroups of patients are more likely to respond to this invasive treatment strategy.

\section{Patient education}

According to the World Health Organization, therapeutic patient education should enable patients to acquire and maintain abilities that allow them to optimally manage their lives with their disease [105]. Education is integrated into the patient's healthcare and must take the person's individual needs into consideration. It is patient-centred and therefore, by definition, a form of personalised medicine, as it takes account of each patient's individuality and specific needs as a person living with a disease. 
Personal asthma education projects are developed in a step-by-step methodology, using the patient's verbatim responses to formulate their problems together with their personal resources and any barriers to tackling these problems [106]. When the problems expressed by patients in their own environment are recontextualised, the healthcare professional necessarily has to construct a tailor-made education project with the patient. For example, if an asthmatic patient has problems taking their asthma medications, a personalised patient education project should first of all endeavour to understand why the patient is not taking the treatment.

The purpose of the education project is to help change the patient's view of and behaviour towards their asthma and their medications. According to the model developed by Tousman et al. [107], behavioural change is dependent on a series of variables related to three more general factors: patient characteristics, the techniques used in the intervention and the structure of the programme. When they are taken into account, the patient-related variables personalise the project, as do the techniques used to encourage adherence to treatment.

Telehealthcare interventions are defined as the remote delivery of healthcare, facilitated by electronic means, involving the exchange of information through personalised interaction between a healthcare professional exercising skills and judgement, and a patient who receives the information. Do telehealthcare tools improve the management of asthmatic patients? A meta-analysis found no evidence for a clinically meaningful impact on the quality of life of asthmatic patients [108]. A more recent meta-analysis published in 2013 examined parallel randomised trials comparing self-management interventions for asthmatic patients delivered via a self-management application on a smartphone versus self-management interventions delivered via traditional methods. Only two studies were analysed, and the results are therefore difficult to interpret [109]. Despite improvements in quality of life and peak expiratory flow in one study, the authors concluded that the current evidence base is insufficient to recommend the use of smartphones for the delivery of self-management interventions.

Internet-based tools have also been analysed in asthma. One study, conducted in 2010, that analysed an internet-based intervention in randomised participants found a small but significant effect on health behaviour, but the largest effect was seen with interventions using behaviour change techniques rather than interventions based on the theory of planned behaviour [110]. This study advocates the use of other, additional methods of communication. A recent review of the literature examining the use of interactive internet-based or mobile device platforms found that these technologies were well perceived, especially in disadvantaged populations and by patients with poorly controlled asthma [111]. Patients with well controlled asthma are less well disposed to these technologies.

In conclusion, patient education is a form of personalised medicine when it is patient-centred and not standardised. Telehealthcare and internet media should be integrated into education projects as a tool, rather than as a standalone intervention. It would be useful to confirm the value of this type of approach in additional studies.

\section{A promising new avenue: upending biomedical concepts}

So far in this article, we have reviewed the various clinical phenotypes, some of which have been recognised as such for decades and that have emerged from the analysis of large cohorts of thoroughly annotated patients. When these cohorts are accompanied by collections of lung or peripheral biosamples, it opens up the possibility of looking for biomarkers specific to a particular clinical phenotype. This search for potential biomarkers can be opened still wider, through genome-wide screening using "omics" technologies, to identify new, biologically defined phenotypes, ideally corresponding to groups of patients that respond to targeted therapies identified through these biological signatures [112].

Basic research conducted over the past 25 years showed that asthma was characterised in most cases by Th2 cell activation, leading to chronic eosinophilic airway inflammation. However, studies on bronchial biopsies and cluster analyses including examination of induced sputum showed that this canonical Th2 inflammation was not present in all asthmatic patients [113]: neutrophils predominate in some forms of asthma, while normal levels of granulocyte populations are found in forms referred to as paucigranulocytic [114]. In addition, eosinophilic inflammation is often accompanied by a neutrophil component [115]. The value of distinguishing between Th2 asthma and non-Th2 asthma is, firstly, that the former is more susceptible to corticosteroid therapy and, secondly, that many targeted therapies, whether currently available (omalizumab) or in the pipeline, are directed against key molecules of Th2 activation (IgE, IL-4, IL-5, IL-13, etc.) [116].

\section{Th2 biomarkers}

The main marker of Th2 inflammation is induced sputum eosinophilia. It is associated with a risk of exacerbations and is a reliable marker of corticosteroid response [117]. However, this investigation is 
difficult to perform in routine practice. In nonsmoking asthmatics, expired nitric oxide is a fairly good indicator of airway eosinophilia but insufficiently reproducible to be used in everyday practice [118]. Blood eosinophilia correlates poorly with sputum eosinophilia and cannot be considered a reliable marker for the Th2 phenotype at the individual-patient level. Genome-wide transcriptome analysis of bronchial cells from unselected asthmatic patients identified a host of genes that are overexpressed in asthma patients, none of which had been previously implicated in the pathophysiology of asthma [119]. Three of the most highly overexpressed, corticosteroid-sensitive and IL-13-dependent genes were investigated in greater detail. The products of these genes also tended to be overexpressed in asthma patients, but in many cases they were not. One of these genes, periostin, seems to be an important marker for response to the anti-IL-13 antibody lebrikizumab [120]. Omics technologies thus homed in on the Th2 phenotype previously identified through pathophysiological hypotheses derived by basic research. But this "bottom-up" approach can also be used to identify new genes involved in asthma and to demonstrate the diverse pathophysiology of this disease, since none of the overexpressed genes identified were found to be overexpressed in all patients.

\section{Non-Th2 biomarkers}

While Th2 activation has been precisely and unambiguously characterised in eosinophilic asthma, no equivalent has been demonstrated in neutrophilic or paucigranulocytic asthma and, as a result, the inflammation in this rarer group is simply defined by what it is not, i.e. non-Th2 inflammation. Other cytokines have been identified in these non-Th2 forms of asthma, such as IL-17, produced by Th17 lymphocytes, but the involvement of these lymphocytes is less clear than that of Th2 cells and they may act together [121]. Recently, a trial to evaluate the efficacy of the anti-IL-17 receptor antibody brodalumab in asthma produced completely negative results, except in a subgroup of patients who were particularly responsive to fast-acting $\beta_{2}$-agonists [97]. The efficacy of brodalumab in this rare subgroup of severe asthmatics could be due to the direct action of IL-17 on airway smooth muscle [122]. Thus, in this case, from a starting point of traditional pathophysiology, a response to a biologic enabled a new highly reversible and IL-17-dependent asthma phenotype to be defined, the biomarker for which is the $\beta_{2}$-agonist reversibility test. The results of clinical trials of brodalumab underline the gap between animals and humans. Promising results obtained in anti-IL-17 studies in mice have not been confirmed in humans [83, 123]. The genetic difference between mice and humans is obvious but it is also difficult to reproduce human complexity in mice, including comorbidities and exposure to a multitude of factors. This demonstrates the limitations of the traditional approach to biomedical research. New strategies are needed to improve our knowledge about asthma.

\section{Discovery strategies: from phenotype biomarkers to risk factor biomarkers}

The strategies discussed in the previous section correspond to a traditional approach in medicine, which starts with the patient, then describes phenotypes and develops targeted therapies based on hypotheses constructed and verified through studies in models. This approach had led to the discovery of numerous treatments but neglects patients who fall outside the hypothesis. This is the case for most "non-Th2" patients. However, we stand at the threshold of a complete transformation in biology and in how we conceptualise medicine, which could offer solutions for these patients and, more generally, a predictive and preventive approach to asthma [124]. The widespread availability of omics technologies at lower cost will enable the elucidation of biological phenotypes defined by multiple and sometimes complex biomarkers (networks of interacting molecules), which in turn, after validation in cohorts and models, will enable trials of new therapies, developed without a pathophysiological hypothesis. This upending of traditional concepts is accompanied by the generation of huge quantities of biological data (phenotype biomarkers), and presupposes the development of powerful and accurate mathematical tools and algorithms capable of integrating and interpreting the data to deliver diagnostic tools capable of predicting precise biological phenotypes related to responses to innovative drugs.

In addition to the variables traditionally considered in asthma, such as sex, weight, smoking status, allergen sensitisation and family history, many other interesting factors could be taken into account in this new strategy. Genetic studies have contributed to our understanding of the complex, polygenic nature of asthma susceptibility. There are so many potentially interesting genes implicated in asthma susceptibility that it would be impossible to describe them all here, but, for instance, the most replicated loci are the ORM1-like 3 gene (ORMDL3) and the neighbouring gasdermin-like genes (GSDMB) [125]. Other susceptibility loci identified include IL33 on chromosome 9p24, IL1RL1/IL18RL1 on 2q12, WDR36/TSLP on 5q22, HLA-DRA and HLA-DQB1 on 6p21, and IL13 on 5q31 [126-129]. The growing interest in the microbiome in asthma could represent another helpful tool in the detection of predisposition to allergic sensitisation and asthma [130]. Similarly, in the past few years, diet has increasingly been regarded as an important issue in asthma. Several studies have demonstrated the benefit of fruit and vegetable consumption in asthma [131]. In contrast, it has been reported that a high-fat diet has a negative effect on 

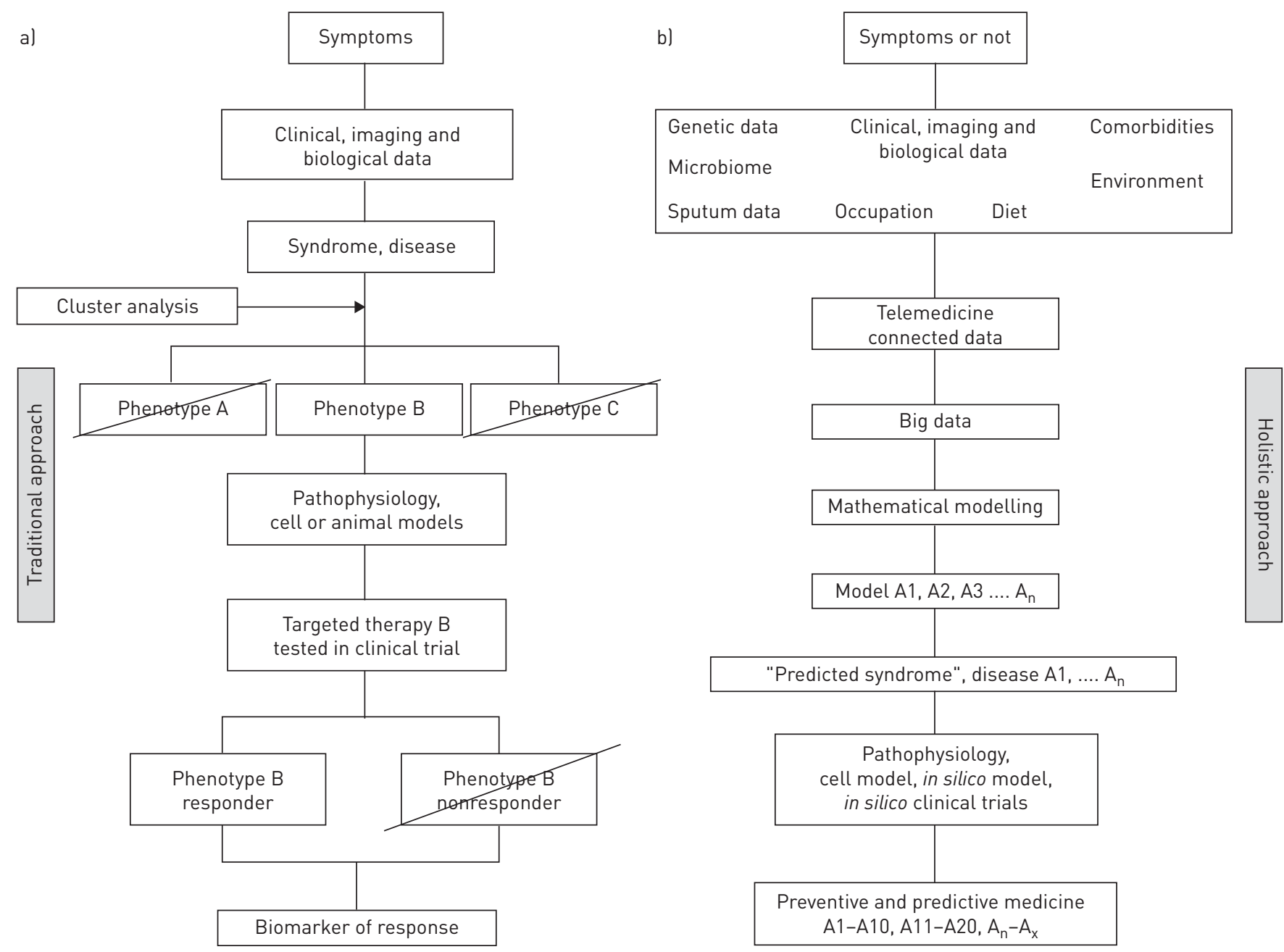

FIGURE 1 Comparison between the a) traditional and b) new holistic approach to biomedical research. The traditional approach starts with observation of symptoms. The symptoms are grouped into syndromes or diseases based on current knowledge of the pathophysiology of the disease. Phenotype B is detected through cluster analysis. Due to the limited number of variables used, phenotypes $\mathrm{A}$ and $\mathrm{C}$ are not detected. Pathophysiological knowledge leads to a focus on the most common, distinctive disease pattern. Cell and animal models are developed in order to identify targeted therapies for phenotype B. This process excludes rarer and less well understood phenotypes. The use of targeted therapy helps refine our understanding of the pathophysiology of phenotype $B$ by identifying responders and nonresponders. Responder and nonresponder biomarkers may be discovered and screened for. In this traditional process, several phenotypes, such as $A, C$ and nonresponders of B, will not benefit from innovative therapies. In summary, this reductionist process starts with symptoms and culminates in laboratory tests. In the holistic process, the subject may be symptomatic or healthy and the analysis of each individual takes into account conventional variables as well as a large body of data including, for instance, their microbiome, genetic data, sputum data, occupation, diet, etc. These data are fed into mathematical algorithms to model the outcome of each subject, generating " $n$ " models, " $n$ " predicted syndromes and " $n$ " "diseases". The pathophysiology will be studied in biological models and in silico models to identify therapies (or management) targeting defined groups (strata) of subjects and to propose preventive, predictive, personalised strategies. In the holistic process, the subject may be healthy, and the "syndrome" is based on biological data.

asthma, particularly in obese patients $[22,132,133]$. These findings illustrate the complexity of the tools required to develop predictive and preventive medicine. This holistic approach would ultimately lead to mathematical modelling of asthma before symptoms develop, providing the opportunity to take action before the disease manifests, through the identification of risk factor biomarkers (figure 1).

\section{Conclusion}

The contributions of both cluster analysis and biological therapies have revealed the possibility of personalised curative medicine for asthma, based on the concept of tailoring management to the individual patient, from diagnosis to therapy and patient education. This personalised approach to medicine, which we could previously only imagine, is already present in our clinical practice. Will things stop there? Probably not. The growing use of omics approaches will enable the discovery of biomarker-defined phenotypes and increase our understanding of asthma pathology. The huge volumes of biological data being generated will no doubt also open up the possibility of personalised preventive medicine, aimed at predicting and 
preventing rather than treating disease, thereby offering individuals the opportunity to take preventive action before asthma develops. Is this the medicine of tomorrow?

\section{References}

1 Wenzel SE, Schwartz LB, Langmack EL, et al. Evidence that severe asthma can be divided pathologically into two inflammatory subtypes with distinct physiologic and clinical characteristics. Am J Respir Crit Care Med 1999; 160: 1001-1008.

2 Britton J. Symptoms and objective measures to define the asthma phenotype. Clin Exp Allergy 1998; 28: Suppl. 1, $2-7$.

Wenzel SE. Asthma: defining of the persistent adult phenotypes. Lancet 2006; 368: 804-813.

Everitt BS, Landau S, Leese M. Cluster Analysis. London, Arnold, 2001.

Hartigan JA. Clustering. Annu Rev Biophys Bioeng 1973; 2: 81-101.

Haldar P, Pavord ID, Shaw DE, et al. Cluster analysis and clinical asthma phenotypes. Am J Respir Crit Care Med 2008; 178: 218-224.

7 Moore WC, Meyers DA, Wenzel SE, et al. Identification of asthma phenotypes using cluster analysis in the Severe Asthma Research Program. Am J Respir Crit Care Med 2010; 181: 315-323.

8 Patrawalla P, Kazeros A, Rogers L, et al. Application of the asthma phenotype algorithm from the Severe Asthma Research Program to an urban population. PLoS One 2012; 7: e44540.

9 Amelink M, de Nijs SB, de Groot JC, et al. Three phenotypes of adult-onset asthma. Allergy 2013; 68: 674-680.

10 Boudier A, Curjuric I, Basagaña X, et al. Ten-year follow-up of cluster-based asthma phenotypes in adults. A pooled analysis of three cohorts. Am J Respir Crit Care Med 2013; 188: 550-560.

11 Romanet-Manent S, Charpin D, Magnan A, et al. Allergic vs nonallergic asthma: what makes the difference? Allergy 2002; 57: 607-613.

12 Virchow JC, Kroegel C, Walker C, et al. Cellular and immunological markers of allergic and intrinsic bronchial asthma. Lung 1994; 172: 313-334.

13 Horn BR, Robin ED, Theodore J, et al. Total eosinophil counts in the management of bronchial asthma. $N$ Engl J Med 1975; 292: 1152-1155.

14 Dweik RA, Boggs PB, Erzurum SC, et al. An official ATS clinical practice guideline: interpretation of exhaled nitric oxide levels (FENO) for clinical applications. Am J Respir Crit Care Med 2011; 184: 602-615.

15 Ford ES. The epidemiology of obesity and asthma. J Allergy Clin Immunol 2005; 115: 897-909.

16 Camargo CA, Weiss ST, Zhang S, et al. Prospective study of body mass index, weight change, and risk of adult-onset asthma in women. Arch Intern Med 1999; 159: 2582-2588.

17 Chen $\mathrm{Y}$, Dales R, Tang M, et al. Obesity may increase the incidence of asthma in women but not in men: longitudinal observations from the Canadian National Population Health Surveys. Am J Epidemiol 2002; 155: 191-197.

18 Farzan S. The asthma phenotype in the obese: distinct or otherwise? J Allergy (Cairo) 2013; 2013: 602908.

19 Holguin F, Bleecker ER, Busse WW, et al. Obesity and asthma: an association modified by age of asthma onset. J Allergy Clin Immunol 2011; 127: 1486-93.e2.

20 Desai D, Newby C, Symon FA, et al. Elevated sputum interleukin-5 and submucosal eosinophilia in obese individuals with severe asthma. Am J Respir Crit Care Med 2013; 188: 657-663.

21 Dixon AE, Poynter ME. Mechanisms of asthma in obesity: pleiotropic aspects of obesity produce distinct asthma phenotypes. Am J Respir Cell Mol Biol 2016; 54: 601-608.

22 Wood LG, Garg ML, Gibson PG. A high-fat challenge increases airway inflammation and impairs bronchodilator recovery in asthma. J Allergy Clin Immunol 2011, 127, 1133-1140.

23 Diaz J, Farzan S. Clinical implications of the obese-asthma phenotypes. Immunol Allergy Clin North Am 2014; 34: 739-751.

24 Agarwal R. Severe asthma with fungal sensitisation. Curr Allergy Asthma Rep 2011; 11: 403-413.

25 Knutsen AP, Vijay HM, Kumar V, et al. Mold sensitivity in children with moderate-severe asthma is associated with HLA-DR and HLA-DQ. Allergy 2010; 65: 1367-1375.

26 Proceedings of the ATS workshop on refractory asthma: current understanding, recommendations, and unanswered questions. American Thoracic Society. Am J Respir Crit Care Med 2000; 162: 2341-2351.

27 Denning DW, Pashley C, Hartl D, et al. Fungal allergy in asthma-state of the art and research needs. Clin Transl Allergy 2014; 4: 14

28 Denning DW, O'Driscoll BR, Hogaboam CM, et al. The link between fungi and severe asthma: a summary of the evidence. Eur Respir J 2006; 27: 615-626.

29 Tillie-Leblond I, Germaud P, Leroyer C. Allergic bronchopulmonary aspergillosis and omalizumab. Allergy 2011; 66: 1254-1256.

30 Chishimba L, Niven RM, Cooley J, et al. Voriconazole and posaconazole improve asthma severity in allergic bronchopulmonary aspergillosis and severe asthma with fungal sensitization. J Asthma 2012; 49: 423-433.

31 McNeill RS, Nairn JR, Millar JS, et al. Exercise-induced asthma. Q J Med 1966; 35: 55-67.

32 Hallstrand TS. New insights into pathogenesis of exercise-induced bronchoconstriction. Curr Opin Allergy Clin Immunol 2012; 12: 42-48.

33 Weiler JM, Bonini S, Coifman R, et al. American Academy of Allergy, Asthma \& Immunology Work Group report: exercise-induced asthma. J Allergy Clin Immunol 2007; 119: 1349-1358.

34 Parsons JP, Hallstrand TS, Mastronarde JG, et al. An official American Thoracic Society clinical practice guideline: exercise-induced bronchoconstriction. Am J Respir Crit Care Med 2013; 187: 1016-1027.

35 Cowan DC, Cowan JO, Palmay R, et al. Effects of steroid therapy on inflammatory cell subtypes in asthma. Thorax 2010; 65: 384-390.

36 Bochenek G, Kuschill-Dziurda J, Szafraniec K, et al. Certain subphenotypes of aspirin-exacerbated respiratory disease distinguished by latent class analysis. J Allergy Clin Immunol 2014; 133: 98-103.

37 Tho NV, Park HY, Nakano Y. Asthma-COPD overlap syndrome (ACOS): a diagnostic challenge. Respirology 2016; 21: 410-418.

38 Gibson PG, McDonald VM. Asthma-COPD overlap 2015: now we are six. Thorax 2015; 70: 683-691. 
Bateman ED, Reddel HK, van Zyl-Smit RN, et al. The asthma-COPD overlap syndrome: towards a revised taxonomy of chronic airways diseases? Lancet Respir Med 2015; 3: 719-728.

Park HW, Kwon HS, Kim TB, et al. Differences between asthma in young and elderly: results from the COREA study. Respir Med 2013; 107: 1509-1514.

Yáñez A, Cho SH, Soriano JB, et al. Asthma in the elderly: what we know and what we have yet to know. World Allergy Organ J 2014; 7: 8 .

Song WJ, Cho SH. Challenges in the management of asthma in the elderly. Allergy Asthma Immunol Res 2015; 7: 431-439.

Sofianou A, Martynenko M, Wolf MS, et al. Asthma beliefs are associated with medication adherence in older asthmatics. J Gen Intern Med 2013; 28: 67-73.

Bourdin A, Molinari N, Vachier I, et al. Prognostic value of cluster analysis of severe asthma phenotypes. J Allergy Clin Immunol 2014; 134: 1043-1050.

Baines KJ, Simpson JL, Wood LG, et al. Transcriptional phenotypes of asthma defined by gene expression profiling of induced sputum samples. J Allergy Clin Immunol 2011; 127: 153-160.

Wenzel SE. Asthma phenotypes: the evolution from clinical to molecular approaches. Nat Med 2012; 18: $716-725$.

Schatz M, Hsu J-WY, Zeiger RS, et al. Phenotypes determined by cluster analysis in severe or difficult-to-treat asthma. J Allergy Clin Immunol 2014; 133: 1549-1556.

Etzel RA. How environmental exposures influence the development and exacerbation of asthma. Pediatrics 2003; 112: 233-239.

Eisner MD. Environmental tobacco smoke exposure and pulmonary function among adults in NHANES III: impact on the general population and adults with current asthma. Environ Health Perspect 2002; 110: 765-770.

Weiss ST, Utell MJ, Samet JM. Environmental tobacco smoke exposure and asthma in adults. Environ Health Perspect 1999; 107: Suppl. 6, 891-895.

Karakaya G, Celebioglu E, Kalyoncu AF. Non-steroidal anti-inflammatory drug hypersensitivity in adults and the factors associated with asthma. Respir Med 2013; 107: 967-974.

Krieger JW, Takaro TK, Song L, et al. The Seattle-King County Healthy Homes Project: a randomized, controlled trial of a community health worker intervention to decrease exposure to indoor asthma triggers. Am J Public Health 2005; 95: 652-659.

Heinzerling L, Mari A, Bergmann K-C, et al. The skin prick test - European standards. Clin Transl Allergy 2013; 3: 3 .

McGarry ME, Thakur N, Oh SS, et al. Effect of obesity on bronchodilator response in minority children with asthma: the GALA II and SAGE II studies. D94. The Bermuda Triangle of pediatric respiratory diseases: asthma, obesity, and sleep disordered breathing. Am J Respir Crit Care Med 2014; 189: A6322.

Barnes PJ, Dweik RA, Gelb AF, et al. Exhaled nitric oxide in pulmonary diseases: a comprehensive review. Chest 2010; 138: 682-692.

Petsky HL, Cates CJ, Lasserson TJ, et al. A systematic review and meta-analysis: tailoring asthma treatment on eosinophilic markers (exhaled nitric oxide or sputum eosinophils). Thorax 2012; 67: 199-208.

Agarwal R, Chakrabarti A, Shah A, et al. Allergic bronchopulmonary aspergillosis: review of literature and proposal of new diagnostic and classification criteria. Clin Exp Allergy 2013; 43: 850-873.

Normansell R, Walker S, Milan SJ, et al. Omalizumab for asthma in adults and children. Cochrane Database Syst Rev 2014; 1: CD003559.

Long A, Rahmaoui A, Rothman KJ, et al. Incidence of malignancy in patients with moderate-to-severe asthma treated with or without omalizumab. J Allergy Clin Immunol 2014; 134: 560-567.

Busse W, Trazskoma B, Omachi T, et al. Evaluating omalizumab persistency of response after long-term therapy (XPORT). Eur Respir J 2014; 44: Suppl. 58, P3485.

Nopp A, Johansson SG, Adédoyin J, et al. After 6 years with Xolair; a 3-year withdrawal follow-up. Allergy 2010; 65: 56-60.

Lai T, Wang S, Xu Z, et al. Long-term efficacy and safety of omalizumab in patients with persistent uncontrolled allergic asthma: a systematic review and meta-analysis. Sci Rep 2015; 5: 8191.

Hanania NA, Alpan O, Hamilos DL, et al. Omalizumab in severe allergic asthma inadequately controlled with standard therapy: a randomized trial. Ann Intern Med 2011; 154: 573-582.

Hanania NA, Wenzel S, Rosén K, et al. Exploring the effects of omalizumab in allergic asthma: an analysis of biomarkers in the EXTRA study. Am J Respir Crit Care Med 2013; 187: 804-811.

Kouro T, Takatsu K. IL-5- and eosinophil-mediated inflammation: from discovery to therapy. Int Immunol 2009; 21: 1303-1309.

O'Byrne PM. The demise of anti Il-5 for asthma, or not. Am J Respir Crit Care Med 2007; 176: 1059-1060.

Leckie MJ, ten Brincke A, Khan J, et al. Effects of an interleukin-5 blocking monoclonal antibody on eosinophils, airway hyper-responsiveness and the late asthmatic response. Lancet 2000; 356: 2144-2148.

Kips JC, O'Connor BJ, Langley SJ, et al. Effect of SCH55700, a humanized anti-human interleukin-5 antibody, in severe persistent asthma: a pilot study. Am J Respir Crit Care Med 2003; 167: 1655-1659.

Flood-Page P, Swenson C, Faiferman I, et al. A study to evaluate safety and efficacy of mepolizumab in patients with moderate persistent asthma. Am J Respir Crit Care Med 2007; 176: 1062-1071.

Nair P, Pizzichini M, Kjarsgaard M, et al. Mepolizumab for prednisone-dependent asthma with sputum eosinophilia. N Engl J Med 2009; 360: 985-993.

Haldar P, Brightling CE, Hargadon B, et al. Mepolizumab and exacerbations of refractory eosinophilic asthma. N Engl J Med 2009; 360: 973-984.

Bel E, Wenzel S, Thompson P, et al. Oral glucocorticoid-sparing effect of mepolizumab in eosinophilic asthma N Engl J Med 2014; 371: 1189-1197.

Pom S, Bleecker E, et al. Mepolizumab for severe eosinophilic asthma (DREAM): a multicentre, double-blind, placebo-controlled trial. Lancet 2012; 380: 651-659.

Ortega HG, Liu MC, Pavord ID, et al. Mepolizumab treatment in patients with severe eosinophilic asthma. N Engl J Med 2014; 371: 1198-1207. 
Castro M, Mathur S, Hargreave P, et al. Reslizumab for poorly controlled eosinophilic asthma: a randomized, placebo-controlled study. Am J Respir Crit Care Med 2011; 184: 1125-1132.

Castro M, Zangrilli J, Wechsler ME, et al. Reslizumab for inadequately controlled asthma with elevated blood eosinophil counts: results from two multicentre, parallel, double-blind, randomised, placebo-controlled, phase 3 trials. Lancet Respir Med 2015; 3: 355-366.

Molfino NA, Gossage D, Lolbeck R, et al. Molecular and clinical rationale for therapeutic targeting of interleukin-5 and its receptor. Clin Exp Allergy 2012; 42: 712-737.

Laviolette M, Gossage DL, Gauvreau G, et al. Effects of benralizumab on airway eosinophils in asthmatic patients with sputum eosinophilia. J Allergy Clin Immunol 2013; 132: 1086-1096.

Castro M, Wenzel SE, Bleecker ER, et al. Benralizumab, an anti-interleukin 5 receptor $\alpha$ monoclonal antibody, versus placebo for uncontrolled eosinophilic asthma: $\mathrm{a}$ phase $2 \mathrm{~b}$ randomised dose-ranging study. Lancet Respir Med 2014; 2: 879-890.

Olin JT, Wechsler ME. Asthma: pathogenesis and novel drugs for treatment. BMJ 2014; 349: g5517.

Wenzel S, Ford L, Pearlman D, et al. Dupilumab in persistent asthma with elevated eosinophil levels. $N$ Engl J Med 2013; 368: 2455-2466.

Beck LA, Thaçi D, Hamilton JD, et al. Dupilumab treatment in adults with moderate-to-severe atopic dermatitis. N Engl J Med 2014; 371: 130-139.

Wenzel S, Wilbraham D, Fuller R, et al. Effect of an interleukin-4 variant on late phase asthmatic response to allergen challenge in asthmatic patients: results of two phase 2a studies. Lancet 2007; 370: 1422-1431.

Corren J, Lemanske RF, Hanania NA, et al. Lebrikizumab treatment in adults with asthma. N Engl J Med 2011; 365: 1088-1098.

Piper E, Brightling C, Niven R, et al. A phase II placebo-controlled study of tralokinumab in moderate-to-severe asthma. Eur Respir J 2013; 41: 330-338.

Teach SJ, Gill MA, Togias A, et al. Preseasonal treatment with either omalizumab or an inhaled corticosteroid boost to prevent fall asthma exacerbations. J Allergy Clin Immunol 2015; 136: 1476-1485.

Dias-Júnior SA, Reis M, de Carvalho-Pinto RM, et al. Effects of weight loss on asthma control in obese patients with severe asthma. Eur Respir J 2014; 43: 1368-1377.

Jensen ME, Gibson PG, Collins CE, et al. Diet-induced weight loss in obese children with asthma: a randomized controlled trial. Clin Exp Allergy 2013; 43: 775-784.

Luna-Pech JA, Torres-Mendoza BM, Luna-Pech JA, et al. Normocaloric diet improves asthma-related quality of life in obese pubertal adolescents. Int Arch Allergy Immunol 2014; 163: 252-258.

Scott HA, Gibson PG, Garg ML, et al. Dietary restriction and exercise improve airway inflammation and clinical outcomes in overweight and obese asthma: a randomized trial. Clin Exp Allergy 2013; 43: 36-49.

Reddy RC, Baptist AP, Fan Z, et al. The effects of bariatric surgery on asthma severity. Obes Surg 2011; 21: 200-206.

Boulet LP, Turcotte H, Martin J, et al. Effect of bariatric surgery on airway response and lung function in obese subjects with asthma. Respir Med 2012; 106: 651-660.

Desai LP, Wu Y, Tepper RS, et al. Mechanical stimuli and IL-13 interact at integrin adhesion complexes to regulate expression of smooth muscle myosin heavy chain in airway smooth muscle tissue. Am J Physiol Lung Cell Mol Physiol 2011; 301: L275-L284.

Busk M, Busk N, Puntenney P, et al. Use of continuous positive airway pressure reduces airway reactivity in adults with asthma. Eur Respir J 2012; 41: 317-322.

Brusselle GG, Vanderstichele C, Jordens P, et al. Azithromycin for prevention of exacerbations in severe asthma (AZISAST): a multicentre randomised double-blind placebo-controlled trial. Thorax 2013; 68: 322-329.

Nair P, Gaga M, Zervas E, et al. Safety and efficacy of a CXCR2 antagonist in patients with severe asthma and sputum neutrophils: a randomized, placebo-controlled clinical trial. Clin Exp Allergy 2012; 42: 1097-1103.

Busse WW, Holgate S, Kerwin E, et al. Randomized, double-blind, placebo-controlled study of brodalumab, a human anti-IL-17 receptor monoclonal antibody, in moderate to severe asthma. Am J Respir Crit Care Med 2013; 188: $1294-1302$.

Bittar HET, Yousem SA, Wenzel SE. Pathobiology of severe asthma. Annu Rev Pathol 2015; 10: 511-545.

Cox PG, Miller J, Mitzner W, et al. Radiofrequency ablation of airway smooth muscle for sustained treatment of asthma: preliminary investigations. Eur Respir J 2004; 24: 659-663.

Bicknell S, Chaudhuri R, Thomson NC. Bronchial thermoplasty in asthma. Breathe 2014; 10: 48-59.

Pretolani M, Dombret MC, Thabut G, et al. Reduction of airway smooth muscle mass by bronchial thermoplasty in patients with severe asthma. Am J Respir Crit Care Med 2014; 190: 1452-1454.

Med 2007; 356: 1327-1337.

3 Castro M, Rubin AS, Laviolette M, et al. Effectiveness and safety of bronchial thermoplasty in the treatment of severe asthma: a multicenter, randomized, double-blind, sham-controlled clinical trial. Am J Respir Crit Care Med 2010; 181: 116-124.

04 Chanez P, Boulet LP, Brillet PY, et al. Bronchial thermoplasty in the treatment of severe adult asthma. Rev Mal Respir 2015; 32: 97-109.

5 World Health Organization. Therapeutic Patient Education: Continuing Education Programmes for Health Care Providers in the Field of Prevention of Chronic Diseases, Report of a WHO Working Group. Copenhagen, WHO Regional Office for Europe, 1998.

06 Morichaud A. Éducation Thérapeutique du Patient: Méthodologie du "Diagnostic Éducatif" au "Projet Personnalisé" Partagés. Aix-en-Provence, Presse Universitaires de Provence, 2014.

Tousman S, Zeitz HJ, Bristol CM. A cognitive behavioral approach to asthma patient education. Adv Manag Respir Care 2002; 11: 47-50.

McLean S, Chandler D, Nurmatov U, et al. Telehealthcare for asthma. Cochrane Database Syst Rev 2010; 10: CD007717.

09 Marcano Belisario JS, Huckvale K, Greenfield G, et al. Smartphone and tablet self management apps for asthma. Cochrane Database Syst Rev 2013; 11: CD010013. 
Webb TL, Joseph J, Yardley L, et al. Using the internet to promote health behavior change: a systematic review and meta-analysis of the impact of theoretical basis, use of behavior change techniques, and mode of delivery on efficacy. J Med Internet Res 2010; 12: e4.

111 Mosnaim GS, Powell LH, Rathkopf M. A review of published studies using interactive Internet tools or mobile devices to improve asthma knowledge or health outcomes. Pediatr Allergy Immunol Pulmonol 2012; 25 : 55-63.

112 Agusti A, Antó JM, Auffray C, et al. Personalized respiratory medicine: exploring the horizon, addressing the issues. Summary of a BRN-AJRCCM workshop held in Barcelona on June 12, 2014. Am J Respir Crit Care Med 2015; 191: 391-401.

113 Wenzel SE, Schwartz LB, Langmack EL, et al. Evidence that severe asthma can be divided pathologically into two inflammatory subtypes with distinct physiologic and clinical characteristics. Am J Respir Crit Care Med 1999; 160: $1001-1008$.

114 Jatakanon A, Uasuf C, Maziak W, et al. Neutrophilic inflammation in severe persistent asthma. Am J Respir Crit Care Med 1999; 160: 1532-1539.

115 Hastie AT, Moore WC, Meyers DA, et al. Analyses of asthma severity phenotypes and inflammatory proteins in subjects stratified by sputum granulocytes. J Allergy Clin Immunol 2010; 125: 1028-1036.

116 Blanc FX, Magnan A. Personalized medicine for asthma: it's now. Rev Mal Respir 2013; 30: 601-604.

117 Green RH, Brightling CE, McKenna S, et al. Asthma exacerbations and sputum eosinophil counts: a randomised controlled trial. Lancet 2002; 360: 1715-1721.

118 Smith AD, Cowan JO, Filsell S, et al. Diagnosing asthma: comparisons between exhaled nitric oxide measurements and conventional tests. Am J Respir Crit Care Med 2004; 169: 473-478.

119 Woodruff PG, Boushey HA, Dolganov GM, et al. Genome-wide profiling identifies epithelial cell genes associated with asthma and with treatment response to corticosteroids. Proc Natl Acad Sci USA 2007; 104: $15858-15863$

120 Corren J, Lemanske RFJr, Hanania NA, et al. Lebrikizumab treatment in adults with asthma. N Engl J Med 2011; 365: 1088-1098.

121 Chesné J, Braza F, Mahay G, et al. IL-17 in severe asthma: where do we stand? Am J Respir Crit Care Med 2014; 190: 1094-1101.

122 Chesné J, Braza F, Chadeuf G, et al. Prime role of IL-17A in neutrophilia and airway smooth muscle contraction in a house dust mite-induced allergic asthma model. J Allergy Clin Immunol 2015; 135: 1643-1645.e5.

123 Park SJ, Lee YC. Interleukin-17 regulation: an attractive therapeutic approach for asthma. Respir Res 2010; 11: 78.

124 Hood L, Balling R, Auffray C. Revolutionizing medicine in the 21st century through systems approaches. Biotechnol J 2012; 7: 992-1001.

125 Ortega VE, Meyers DA. Implications of population structure and ancestry on asthma genetic studies. Curr Opin Allergy Clin Immunol 2014; 14: 381-389.

126 Moffatt MF, Gut IG, Demenais F, et al. A large-scale, consortium-based genomewide association study of asthma. N Engl J Med 2010; 363: 1211-1221.

127 Torgerson DG, Ampleford EJ, Chiu GY, et al. Meta-analysis of genome-wide association studies of asthma in ethnically diverse North American populations. Nat Genet 2011; 43: 887-892.

128 Li X, Howard TD, Zheng SL, et al. Genome-wide association study of asthma identifies RAD50-IL13 and HLA-DR/DQ regions. J Allergy Clin Immunol 2010; 125: 328-335.

129 Li X, Ampleford EJ, Howard TD, et al. Genome-wide association studies of asthma indicate opposite immunopathogenesis direction from autoimmune diseases. J Allergy Clin Immunol 2012; 130: 861-868.

130 Huang YJ, Boushey HA. The microbiome in asthma. J Allergy Clin Immunol 2015; 135: 25-30.

131 Garcia-Larsen V, Del Giacco SR, Moreira A, et al. Asthma and dietary intake: an overview of systematic reviews. Allergy 2016; 71: 433-442.

132 Varraso R, Kauffmann F, Leynaert B, et al. Dietary patterns and asthma in the E3N study. Eur Respir J 2009; 33: 33-41.

133 Rosenkranz RR, Rosenkranz SK, Neessen KJ. Dietary factors associated with lifetime asthma or hayfever diagnosis in Australian middle-aged and older adults: a cross-sectional study. Nutr J 2012; 11: 84-89. 